\title{
EVALUACIÓN DE EXPLOTACIÓN DE METAL X-KERNEL V.3.0.3
}

\section{Antonio Barreñada García}

\begin{abstract}
Starting from a brief historical introduction and short description we have attempted to put forward an assessment of the running, performance and development of the Machine Translation system METAL X-Kernel v. 3.0.3, in the UNIX (Sparc Station IPX), enviroment. Our assessment has been carried out within the "Servicio de Traducción Científico-Técnica de la Universidad de León, España" over a period of one year of experience in MT. The translations, from English into Spanish, have been restricted to morphological and semantic procedures with no interpretative application. Texts from various fields (biology, veterinary science, sport medicine, etc.) have been dealt with and special emphasis has been put on the specific vocabulary of phonetics. It has been done from the point of view of a linguist user taking into account the suitability of the tool.
\end{abstract}

La idea, el concepto de TA (Traducción Automática) podría comprender desde la restringida referencia a "aquel tipo de traducción que realiza una máquina sin intervención alguna del hombre, exceptuando, claro está, las labores de preedición y postedición" (Slocum, 1985, citado por P.Fernández Nistal, 1994, p.135) hasta la menos restrictiva y más reciente tendencia que admite "cualquier traducción realizada por ordenador con o sin intervención humana" (Ibid, que cita Newton, 1992, Balkan, 1992, Tapias-Merino y Siles Sánchez, 1992).

Según Hutchins, "hoy día, el término inglés Machine Translation (MT) es la denominación tradicional y ya consagrada de los sistemas informáticos que llevan a cabo traducciones de una lengua a otra, con o sin intervención humana. Otras denominaciones anteriores tales como 'Mechanical Translation' y 'Automatic Translation' rara vez se usan ya en inglés,..." (Hutchins, 1992[1995], p.27).

Tanto para el propio Hutchins como para la mayoría de los especialistas en el tema, los límites entre THAO (Traducción Humana Asistida por Ordenador) -“MAHT (MachineAided Human Translation)- y TAAH (Traducción Automática Asistida por el Hombre -"HAMT (Human-Aided Machine Translation)"- los límites, decíamos, resultan inciertos. Ambas pueden caer bajo un epígrafe genérico nombrado TAO (Traducción Asistida por Ordenador). Y esto, como podremos ver, es algo más que una cuestión terminológica.

El empleo de máquinas computadoras, de ordenadores, con aplicaciones en la traducción se considera deudor "una vez más" de las ocupaciones belicistas. Lá Guerra Fría conoce el interés por la traducción de mensajes del ruso al inglés (e instituciones como 
Army, Navy, USAF, CIA y NSA son patrocinadoras de las investigaciones y Memorandum). En este marco aparecen los trabajos e informes de Warren Weaver (15 de julio de 1949, Locke y Booth eds., 1955, pp. 15-22), génesis comúnmente aceptada de la TA, así como la contribución de Andrew D. Booth.

A comienzos de los años 60 aparece una sección experimental en la Universidad de Texas "que no se venía ocupando de la TA sino de la CALL(Computer Assisted Language Learning)" dirigida por D.C. Travis (del Departamento de Germanística) y W. Holtzmann (del Dep. de Sociología). A mediados de esa década Lehmann y Pedergraft informan de que el grupo de TA de la U. de Texas está utilizando programas para verificar datos sintácticos del inglés, alemán, ruso y chino.

La USAF "encargó" al Linguistics Research Center $(L R C)$ de esa Universidad de Texas (Fundado en 1961), bajo la dirección de Wifred Lehmann, el desarrollo de un sistema de TA del que va a ser núcleo traductor el programa METAL (Mechanical Translation and Analysis of Language).

Después del estrangulamiento del causado por el informe ALPAC el LRC sigue funcionando. A partir de 1978 el Rome Air Development Center sufraga la aplicación de los hallazgos teóricos del Centro.

La historia de METAL nos lleva desde una primera fase de investigación lingüística (desarrollada en los 60) centrada en el desarrollo de un sistema de transferencia bidireccional "con reglas de transferencia sintáctica reversibles" hasta 1968.

"En aquel momento, la mayor parte de la comunidad científica dedicada a la investigación sobre TA compartía la opinión de que las mejores expectativas para el logro de avances significativos residían en el desarrollo de programas de transferencia. Los investigadores del LRC (Linguistic Research Center) en Austin, Texas (uno de los pocos en que se continuó investigando sobre TA después del informe ALPAC) habían llegado a conclusiones similares después de experimentar con un programa interlingüe, por lo que dirigieron sus esfuerzos al desarrollo del programa METAL basado en la transferencia;".

(Hutchins, 1992 [1995], p. 33)

El sistema corre sobre un CDC 6600 en FORTRAN. No se soporta multitarea y mínimamente se emula un proceso interactivo.

Entre los años 1970 y 1975 se desarrolla una segunda fase de investigación con enfoque interlingual y trabajando "aún" sobre el par alemán-inglés.

Esta fase se inicia con una conferencia deliberativa que el LRC convoca para reconsiderar ALPAC.

En 1978 comenzaba el apoyo financiero de la empresa SIEMENS A.G. de Munich. En palabras de Thomas Schneider, cualificado miembro de la Compañía:

"In an attempt to solve this in-house problem, Siemens became involved in the area of machine translation. After negative experiences with commercially available MT systems, a decision was made to start a research and development project. Its goal was to build an operative 
machine translation system which would be able to increase the productivity of the in-house translators and reduce turn-around time, i.e. the time span from receipt ot a source document to delivery of the target document.

In 1978 Siemens entered into a cooperative agreement with the University of Texas at Austin. The Linguistics Research Center at UT was in the forunate position of having been able to devote many years to research to this field. The work was conducted under the title of "METAL", and even though the present system bears little resemblance to the early versions, the name has been retained. A first prototype was presented in 1979.

However, it has taken ten long years of development before METAL finally reached the status of a product."

(Schneider, 1992, p.586)

A partir de 1980 SIEMENS se convierte en el único financiador. No puede obviarse la "prehistoria" del prototipo, su vinculación con un Departamento de Germanística y todo el trabajo realizado en análisis léxico-gramatical del par alemán-inglés.

El sistema que la SIEMENS "adquiere" se implementa sobre máquinas LISP Simbolics; su software para el proceso de texto está escrito en SNOBOL y el resto en LISP.

Se ha consegudio una aceleración de los procesos y el trabajo se optimiza trabajando en red con microprocesadores en preedición y postedición.

El diseño de interlingua se cambia al de transferencia (fundamentalmente) y se combina, en un entramado complejo, con el uso de bases de datos como TEAM y servicios de edición.

La tipología del sistema sería la de un híbrido entre los generalmente considerados tipos de interlengua y transferencia:



Por 1985 (según la evaluación in-house de Bennett y Slocum), después de traducir 1000 páginas de texto alemán al inglés, se informa que se ha conseguido entre un 45 y un $85 \%$ de traducciones correctas.

En 1989 sale al mercado el producto METAL: trabaja con el par alemán-inglés sobre plataforma Lisp Simbolics de la serie 36, que se complementan "para la realización de labores de preedición y postedición" con estaciones Siemens MX-2, MX-300 y MX-500 basadas en SINIX. 
La versión del '89 introduce importantes innovaciones:

- Pattern Matching

- Version Control

- Terminology \& Style Checking

- Automated Accounting

- Interfaces to a Terminology data base

En 1994 se publica en META [Vol. 39, n• 1, (p.5-300), pp. 206-212] un informe de F. DEPREZ y otros tres investigadores del Centro Siemens-Nixdorf de Lieja relatando la experiencia con METAL en el Ministerio del Interior Belga con respecto a los pares holandés-francés, francés-holandés.

Es uno de los análisis de funcionamiento más sistemáticos realizados hasta la fecha, si bien no se deslinda de los planteamientos in-house. Se empleó el "Sisyphus toolkit" (procedimiento evaluativo de la compañía productora).

En la evaluación de los textos no preelaborados (reales) se detectó uno de los problemas más significativos del sistema:

"Translation quality and speed within METAL tend to decrease considerably for sentences longer than 20 words."(DEPREZ el al, op. cit., p. 208)

En el año de la publicación de este informe (1994) adquiría el Servicio de Traducción Científico-Técnica de la Universidad de León el sistema, producto, METAL (v. 3.0.3), portado sobre una plataforma SUN con sistema UNIX SVR4. Esta presentación era propicia a su utilización en red, con una configuración apta para que múltiples usuarios se conviertan en potenciales "cotraductores". Sin embargo, la fórmula que se adoptó, inicialmente, en la Universidad de León no fue ésta, sino la de trabajo aislado de la estación..

Para el par de lenguas con que trabaja nuestro equipo (inglés-español) contamos con tres léxicos que ejemplificaremos con la entrada "shimmer":

- monolingüe inglés (SL)

- monolingüe español (TL)

- bilingüe, transfer, que conecta SL y TL

Cada entrada contiene información morfológica, semántica y sintáctica asociada a ella en forma de pares rasgo-valor del tipo: 


\begin{tabular}{|c|c|c|c|}
\hline ALO & (alomorfo) & "shimmer" & \\
\hline CL & (clase morfológica) & (S-01) & (c. léxico-morfol.) \\
\hline CAN & (forma canónica) & "shimmer" & \\
\hline CAT & (categoría sintáctica) & NST & (stem nominal) \\
\hline DR & (requiere determin.) & PR RD & (sí tras "as"; no PP) \\
\hline FUNC & (función sintáctica) & HEAD & \\
\hline KN & (Kind of noun) & CNT & (contable) \\
\hline SNV & (special number) & SGT & (singular sólo) \\
\hline
\end{tabular}

Se consideran categorías morfológicas como:

\begin{tabular}{|l|l|}
\hline 66 66 & "espúrea" \\
\hline DET & determinante \\
\hline AST & raíz adjetival \\
\hline TOKEN & "símbolo"."signo" \\
\hline NST & raíz nominal \\
\hline VST & raíz verbal \\
\hline V-FLEX & flexión verbal \\
\hline N-FLEX & flexión nominal \\
\hline ADV & adverbio \\
\hline VAD & pertícula verbal \\
\hline
\end{tabular}

El léxico bilingüe contiene:

\begin{tabular}{|l|l|l|}
\hline " “X " "L & $\begin{array}{l}\text { especificación de la entrada palabra y } \\
\text { categoría fuente y destino }\end{array}$ & $\begin{array}{l}\text { "shimmer"NST } \\
\text { "reflejo"NST }\end{array}$ \\
\hline tests & $\begin{array}{l}\text { tests opcionales de aplicación de entrada } \\
\text { (suelen fundarse en la existencia o naturaleza } \\
\text { de satélites del verbo) }\end{array}$ & $\begin{array}{l}\text { Prf. S.0.0.00 Tag } \\
\text { (G.V.) }\end{array}$ \\
\hline transformaciones & cambios léxico-estructurales & $\begin{array}{l}\text { <Fb52 FB52 Clara } \\
\text { 30 Jan 92 > }\end{array}$ \\
\hline $\begin{array}{l}\text { datos } \\
\text { introducción }\end{array}$ & (lugar, autor, fecha) & \\
\hline
\end{tabular}

Las gramáticas integran, junto con los léxicos, el componente lingüístico. La gramática de análisis (construída entre otros, sobre formalismos del modelo X barra) se compone de unas 600 reglas de estructura de frase mixtificadas con rasgos léxico-contextuales. Es un sistema abierto a incrementos y modificaciones. 
El analizador sintáctico es un "chart-parser" de isla ascendente que actúa con un procesamiento en paralelo. (Más detalles sobre aspectos lingüísticos y computacionales: THURMAIR 1990a y 1990b.)

La información léxica se organiza de forma jerárquica, en niveles. Todas las formas de una palabra se subyugan a una única entrada de la misma, con llamadas internas a tablas y reglas que permiten generar formas plenas.

A su vez la organización jerárquica se aplica a módulos para los contenidos terminológicos:

\section{FW}

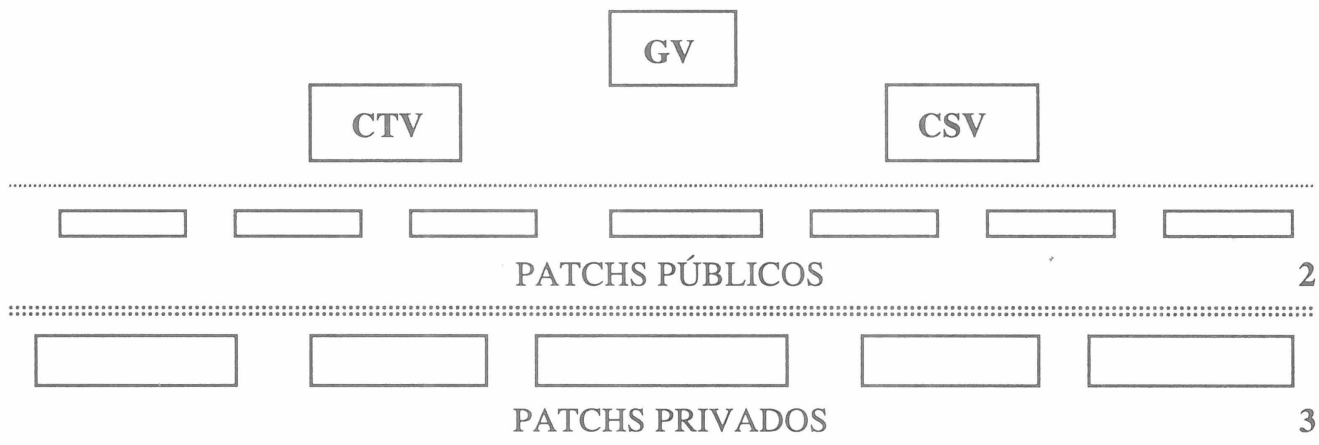

Donde:

$$
\begin{aligned}
& \text { FW }=\text { Palabras-funciones (preposiciones, determinantes, conjunciones) } \\
& \text { GV }=\text { Vocabulario General } \\
& \text { CTV = Vocabulario Técnico Común } \\
& \text { CSV }=\text { Vocabulario Social/administartivo común }
\end{aligned}
$$

Los módulos de los grupos 1 y 2 forman parte del "océano", de la progaramación de acceso automático. Los módulos contenidos en el apartado 3 han de ser solicitados por el usuario, o bien, desembocados al océano en una sesión de volcado; su provisionalidad presenta esa pequeña desventaja junto al gran acierto consistente en realizar una labor de filtrado de los elementos que se pretenden incorporar al sistema.

La versión con que contamos contenía inicialmente un volumen de unas 100.000 entradas en el léxico inglés, 25.000 en el español y 25.000 en el transfer.

La incorporación de nuevos elementos se realiza mediante la "joya" del interface de usuario: el INTERCODER. En valoraciones de SIEMENS, se ha demostrado que su utilización reduce el tiempo de codificado en un producto de factor 10. Las palabras se identifican por su forma canónia y su categoría. 
Ha de observarse que el nuestro es un "minifundio", una explotación "subsistencial" más que "industrial": en el ejercicio 95/96 METAL se utilizó en la traducción de 357 páginas. Ello ha de tenerse en cuenta en toda valoración.

METAL se nos presentaba aplicable para traducir textos técnicos (Bids, descripciones técnicas, manuales de instrucciones, manuales de mantenimiento, ...) y tal ha sido su destino

Si damos por válida una definición del tipo

“Traducir consiste en reproducir en el lenguaje receptor el equivalente más próximo de la lengua de partida, en primer lugar en términos de sentido, en segundo lugar, en términos de estilo." (E.NIDA y CH. TABER, The theory and practice of Translation, Leyden, E.J.Brill, 1974, p.12)

y si tenemos la "honradez" de admitir que

"The state of the art in computational lingüístics does no permit the perfect translation"

(SCHNEIDER, op. cit., p. 591)

podremos contar con una predefinición del marco en el que se puede efectuar una valoración de METAL "referida a nuestra mínima experiencia" como sistema de TA.

Las reglas "siquiera la metodología" para el análisis valorativo de los procedimientos de TA no han pasado a formar parte de un corpus de libre disposición para los usuarios. Ni siquiera para los investigadores.

Aquí, como en otros campos de la LC, la carencia de estandares es uno de los problemas endémicos.

En unas jornadas centradas en la problemática de estandarización celebradas en la Universidad de Deusto en septiembre del 95 por la SEPLN, Marta Sáiz, joven investigadora bilbaína de la UMIST, presentaba una elaborada comunicación sobre "Evaluación en MT" en la que manifestaba:

"Despite forty years of research on MT there is still not a generally accepted, satisfactory an comprehensive evaluation methodology. One might say that the MT problem is also still not well understood, however one should still be able to set up evaluation methodologies.[...]there should be a general evaluation methodology for all NLP applications or whether differences between tasks and applications should be considered leading to different methodologies; which is the object o MT evaluation, and whether evaluation should be black box or glass box."

(M. SÁIZ, "Issues and Approaches in NLP evaluation", en SEPLN, vol 17, pp. 289-290)

Incidiendo en la carencia, M. Sáiz recoge las propuestas más significativas desarrolladas hasta la fecha, desde el contraefectivo informe ALPAC (1996), la evaluación TAUM (1986), la evaluación de SYSTRAN por las USAF (1970-80), hasta el grupo de evaluación 
EAGLES (cuyos resultados se publicaron en 1994), que representa ya una propuesta metodologicamente armonizadora.

Partiendo de la opción por el análisis black box, tipo propio a un usuario no cualificado -como es nuestro caso- (donde el sistema se considera como un todo del cual accedemos tan sólo al input y al output) o una glass box (donde el evaluador tiene acceso a todas las funciones internas del sistema y puede trabajar sobre resultados intermedios), y, preestableciendo de forma sistemática la definición de calidad y error counting, "which is the amount of work needed to correct MT output to make it acceptable as a translation" (Ibid), llega Sáiz a deslindar diversos procedimientos evaluativos que nos van a servir de guía.

Damos por cierta la observación de Hutchins (1992[1995]. p. 25) de que "los principales obstáculos a la hora de traducir por ordenador no han sido ni son informáticos sino lingüísticos. Son problemas tales como la ambigüedad léxica, la complejidad sintáctica, las diferencias de vocabulario entre lenguas, las construcciones elípticas o incorrectas gramaticalmente...", y nos centraremos, pues, en el devenir lingüístico de la traducción.

\section{Evaluación objetiva de Output}

Cómputo de errores: A partir de un texto "real" (223.TXT), parte de un manual de manejo de un programa de análisis de voz, integrado por 10 páginas (221 frases), utilizando los diccionarios genérico y específico propicios, con una ejecución de la fase de preanálisis ( $\sin$ integrar nueva información en el sistema) considerando error todo elemento traducido carente de sentido, observamos 39 errores.

\section{Clasificación de errores:}

Tipo léxico: No es fácil deslindar la causa léxica de la gramatical: suelen presentarse agramaticalidades cuando ocurren errores léxicos, no obstante, parecen claros 22 casos, con dominancia de los producidos por ambigüedad léxica.

$$
\begin{aligned}
\text { ej.: "relative" } & =\text { familiar por relativa } \\
\text { "pitch" } & =\text { lanzar por tono (en contexto no claro de NST) } \\
\text { "short" } & =\text { copa } \text { por corto }
\end{aligned}
$$

La jerarquización de módulos léxicos permite que opciones genéricas (incluso "engendros" para el sueño de la razón) sean preferidas a las específicas que no obstante sí son conocidas por el sistema

$$
\text { ej.: "formant" = forma de hormiga, por formante }
$$

Tipo gramatical: (17) Abundan en los dominios verbales:

- No parece resuelta la traducción del subjuntivo ni las nominalizaciones del gerundio.

- Aparentemente, la simplicidad de una regla del tipo "NST en comienzo de frase debe ser transferido al español con determinante" no siempre es útil. Frecuentemente produce errores nominalizadores. 
- Los movimientos de cadenas de adjetivos con un nombre obedecen a estructuras excesivamente rígidas o uniformes.

\section{Dificultad de resolución:}

- Especial entorpecimiento con los compuestos (.COM); basta con observar su abundancia, si bien, suelen resolverse satisfactoriamente.

- No se plantean soluciones fáciles a los procesos de movimiento (cabría pensar en el basamento generativo de los 70 "en las vacilaciones entorno a las reglas de movimiento y su entidad"). Nos tememos, sin embargo, que en este apartado la desviación del modelo gramatical ha sido mayor hacia soluciones coyunturales vía "contexto".

- El conflicto phrasal verbs /VST + P no parece superado generalmente.

- A pesar del tiempo transcurrido, la dificultad observada en su día en la evaluación del Ministerio Belga del Interior se mantiene: La traducción se hace más dificultosa cuanto más extensa es la frase, resultando que los procesos de subordinación heredan los daños causados por una inicial mala colocación de su cabeza verbal.

\section{Evaluación con Test Suites}

Las pruebas que hemos realizado nos han suministrado conclusiones que, desde lo anecdótico (Después de casi 40 años la frase-test de Bar-Hillel (1959) sigue sin resolverse satisfactoriamente:

"Little John was looking for his toy box. The box was in the pen. John was very happy."

\section{Traducida por METAL:}

"Poco John estaba buscando su caja de juguete. La caja estaba en la pluma. El John era muy feliz." ) deesde la anécdota, decíamos, nos han conducido a planteamientos de mayor formalidad:

Sí son fácilmente solucionadas las ambigüedades cuando la proximidad -la simultaneidad incluso- en el decurso permite colocar a cada entrada en el hueco de su categoría (que no función):

"You can also monitor the input lights on the front panel of the CSL to make sure that the OVER light does not light:

1 interpret.

597 phrases

348 rejected

17.237 seconds

transfer + gener $=12.436$ seconds

"Usted puede controlar también las luces de respuesta (entrada) en la parte delantera del panel del CSL para asegurarse de que la luz de POR ENCIMA no enciende."

Sí hemos observado la perfecta resolución de anáforas (incluso, en ocasiones, con correcta adecuación estilística a la salida en español). 
Sí hemos observado la resolución de elisiones para la categoría nominal, pero no tanto para el verbo.

Hemos apreciado que la ambigüedad no gana en su resolución con una organización jerárquica de lexicones y que, aparentemente, su solución ha sido planteada con una acumulación de información contextual que no siempre es efectiva;

Y hemos creído ver que, respecto al asunto de las nominalizaciones, el planteamiento conforme a la gramática de los 70 (subyacente en el modelo Xbarra) "tal que que N y V figuren en una misma entrada léxica con dos especificaciones de rasgos vinculados" no es resolutivo, y no lo es más desde el momento en que se ve supeditado a procedimientos de análisis "sintáctico" como focalizar toda estructura en el elemento V sin clara salida para su posible elisión.

Una de las "grandezas" consideradas en METAL es su capacidad de realizar análisis y traducciones "válidas" pese al desconocimiento de alguno de los términos integrantes de la frase. Esta consideración puede relativizarse teniendo en cuenta lo ya observado respecto a la implicación léxico-gramatical: La agramaticalidad ( la no validez) de la traducción obedece frecuentemente a una mala colocación del elemento anómalo en la estructura del análisis.

\section{Evaluación con Test Corpora.}

Dado que no han sido especialmente preelaborados nuestros test suites, no muy distinta es la naturaleza y valoración consiguiente de los Tests Corpora.

Ahora bien, lo que sí es constatable en los textos "reales", y es digno de consideración, es su deplorable calidad.

El probable redactor puede haber sido un ingeniero (cuya L1 fuera el inglés o no) o un "redactor técnico" (escritor especializado en redacciones técnicas, cuyo texto está mejor escrito pero técnicamente peor cualificado). En cualquier caso son significativos:

- la omnipresencia de siglas

- la ausencia de coordinaciones

- la abundancia de numeraciones

- los usos neologistas fuera de toda norma y en ocasiones muy particulares de la empresa manufacturadora del producto.

Y todo ello ha de redundar, lamentablemente, en la calidad de la traducción, sin que ello pueda ser imputable al sistema de TA.

En principio cabe pensar que pudo haberse trabajado en el desarrollo de METAL de cara a los textos técnicos en la creencia de su sistematicidad formal, léxica y gramatical. Sin embargo, al enmfrentarse con este tipo de textos cabe echar de menos las más estables formas incluso de textos poéticos.

Ya en 1982 los desarrolladores de METAL estaban seguros de que era un sistema viable: 
"It could translate, it had an user interface, and it could deformat and reformat certain document Types."

(SCHNEIDER,op. cit. ,p. 592)

Examinados en forma in house los fallos del sistema se desarrollaron herramientas y procedimientos para solventarlos (p.ej.: MPM) que en ocasiones (y el ejemplo dado puede ser válido) están más próximos a la solución coyuntural que a un planteamiento sistemático.

El usuario es, por desgracia, un entusiasta de tercera generación: su ilusión con respecto a un producto sucede a la original de sus creadores y a la intermedia de sus distribuidores.

Cuando la emoción del creador decae puede reanimarse con el sustento institucionalpúblico ( el Ministerio de Industria español subvencionó ocasionalmente METAL, así como AENOR, en la pretensión de progresar en una normalización terminológica) o la interesada participación del mercader, mientras dure. Nuestras facultades saben mucho de proyectos abandonados ante la desapetencia de los capitales.

Cuando el beneficio empresarial no aparece, tan sólo pueden soportar investigación y desarrollo las muy poderosas firmas. Incluso éstas llegan a una situación límite de sontenibilidad de un proyecto y adoptan su abandono o reconversión.

Los vientos en el mundo de la traducción soplan con fuerza hacia la pragmática consideración de la TAHO frente a la TAAH. Sea: parece existir un nuevo declive en el interés por la TA como "absoluto", virando hacia meros sistemas de apoyo.

En el próximo futuro de METAL está el abandono "temporal" de la actual línea de desarrollo. El actual productor, GMS, en colaboración con la editorial Langenscheidt, pone de forma inmediata a la venta un subproducto (T1) para plataforma PC en dos versiones (básica y profesional) "los pares español-inglés e inglés-español estarán disponibles el próximo año" a un precio competitivo con productos del tipo del Power Translator, postergando para un medio plazo el desarrollo del producto ahora presentado en sistema UNIX.

La versión sobre PC se dice similar a METAL en su sistema (análisis-transfergeneración) y se dota de similares herramientas (intercoder) si bien simplificadas. Tan sólo se modifica sustancialmente el núcleo, el motor del sistema.

Para la elaboración del producto PC ha sido fundamental el desarrollo de diccionarios realizado en la explotación de METAL.

La consideración de que un notabilísimo desarrollo científico-tecnológico puede ser excesivo (y por ello inadecuado) a la tarea que se le destina, nos lleva, por último, a quedarnos con la duda de si no ha sido mucho CAT para estos pequeños roedores de libros. 


\section{BIBLIOGRAFÍA}

ALEXA, M., (1993), Corpus-based Sublanguage Analysis for a Multilingual Generation Systems,

ALPAC (Automatic Language Processing Advisory Committee), (1966), Computers in translation and Linguistics, US National Academic of Sciences, Sciences National Research Council.

ARNOLD, D. et al, (1994) Machine Translation: An Introductory Guide, Cambridge (Mass.)-Oxford (Eng.), Blackwell pub.

ARNOLD, D., SADLER, L. \& HUMPRHEYS, R.L., "Evaluation: An assesment", Machine Translation, vol 8, nn. 1-2, Amsterdam, Kluwer Academic Publishers.

DEPREZ, F., ADRIAENS, G., DEPOORTERE, B. Y BRAEKELEER, G. de, (1994), "Experiences with METAL at the Belgian Minietry of the Interior", META, Outremont, PQ, Cnada, 39:1, Mar 1994, pp. 206- 212.

EAGLES Evaluation Working Group, (1994) Report of the Evaluation of NLP Systems Working Group, Pisa, Consorcio Pisa Richerche, Draft Interim Report.

FERNÁNDEZ NISTAL, Purificación, (1994), "Introducción 'a la traducción automática", ASPECTOS de la traducción inglés-español: $2^{\circ}$ curso superior de traducción, Valladolid.

FONTENELLE, T., ADRIAENS, G., \& BRAEKELEER, G. de, (1994),'The Lexical Unit in the METAL\$2f MT System", Machine Translation, Dordrecht, Netherlands, 9:1, pp 1-19.

HOUSE, Juliane, (1981), A Model for Translation Quality Assesment, Tubingen, Gunter Narr Verlag.

HUTCHINS, W John \& SOMERS, Harold L., (1992), An Introduction to Machine Translation, Cambridge, Academis Press Limited, ‘[1995] (trad) Joseba K. Abaitua (Ed.), Introducción a la Traducción Automática, Madrid, Visor.

HUTCHINS, W.J., (1986), Machine Translation: Past, Present, Future, Ellis Horwood/Wiley, New York.

KING, M. \& FALKEDAL, K., (1990), "Using Test Suites in the Evaluation of Machine Translation Systems”, Proceedings of Coling ë90, Helsinki, pp. 211-219.

KING, M. (Ed.), (1987), Machine Translation Today: The State of the Art, Proceedings of the 3rd. Lugano Tutorial, Lugano, Switzerland, 2-7 April, 1984, Edimburg, E.U.P..

LAMIROY, B. \& GEBRUERS, R., (1989), "Syntax and Machine Translation: The METAL Project”, Lingvisticae-Investigationes, Amsterdam, 13:2, pp. 307-332.

LITTLE, P., (1990), "METAL: Machine translation in Practice", C.Picken (Ed..) Translating and the computer II, London, ASLIB. 
LIU, J. \& LIRO, J., (1987), "The METAL English-to-German System: First Report", Computers-and-Translation, Dordrecht, Nederlands, 1987 Oct.-Dec., pp. 205-218

PALMER, H., \& FININ, T., (1990), "workshop of the Evaluation of Natural Language Processing Systems", Computational Linguistics, vol. 16, n 3, sep. 1990.

PUGH, J., (1992) "The Story so far: an Evaluation of Machine Translation in the World Today", Newton, J., (Ed.) Computers in Translation. A practical Appraisal, Routledge, pp. 14-32.

REGALES SERNA, Antonio, (1983), La traducción automática, tesis doctoral presentada en la Universidad de Santiago de Compostela en 1976, Oviedo, Pentalfa, 1983 (microficha)

ROBAYO GARCÍA, M., (1978), Un generador de analizadores sintácticos para lenguajes $L L(1)$ expresados en SRL, tesis y disertaciones académicas, Universidad de Sevilla.

SÁIZ, M., (1995), "Issues and Approaches in NLP Evaluation", en Procesamiento del Lenguaje Natural, Actas de la SEPLN, revista n 17, septiembre de 1995.

SCHNEIDER, T., (1990), "METAL: An Operative Machine Translation System", P. McKay (Ed.), Computers and the Arabic Language, New York, Hemisphere.

SCHNEIDER, T., (1992), "User Driven Development: METAL as an Integrated Multilingual System”, META, Outremont, PQ, Canada, Dec. 1992, 37:4, pp. 583-594.

SIETEC/INCYTA, (1994), Manual METAL/X KERNEL, V.3.0.3.

SLOCUM, J., (1984), "METAL: The LRC Machine Translation System", Proceedings of 3rd. Lugano Tutorial, Lugano, Switzerland, 2-7 Apr. 1984, M. King. (Ed.), Machine Translation Today: The State of the Art, Edinburgh, EUP, 1987.

SUMMIT, (1991), Proceedings of MT Summit III, Washington DC, pp. 141-146.

THURMAIR, G., (1990a), "METAL, Computer Integrated Translation”, J. McNaught (Ed.), Proceedings of the SALT workshop 1990, Manchester.

THURMAIR, G., (1990b), "Complex Lexical Transfer in METAL”, Proceedings of the 3rd. International Conference on Theoretical and Methodological Issues in Machine Translation of Natural Language, University of Texas at Austin.

WHITE, J.S., (1987), "The Research Enviroment in The METAL Project"; Sponsored by Assn. for Computational Ling., S. Niremburg (Ed..), Machine Translation: Theoretical and Methodological Issues, Cambridge, CUP, pp. 225-246. 
